\title{
Molecular alterations of coexisting thyroid papillary carcinoma and anaplastic carcinoma: identification of TERT mutation as an independent risk factor for transformation
}

Naoki Oishi $^{1}$, Tetsuo Kondo ${ }^{1}$, Aya Ebina ${ }^{2}$, Yukiko Sato ${ }^{3}$, Junko Akaishi ${ }^{4}$, Rumi Hino ${ }^{3}$, Noriko Yamamoto ${ }^{3}$, Kunio Mochizuki ${ }^{1}$, Tadao Nakazawa ${ }^{1}$, Hiroshi Yokomichi ${ }^{5}$, Koichi Ito ${ }^{4}$, Yuichi Ishikawa ${ }^{3}$ and Ryohei Katoh ${ }^{1}$

${ }^{1}$ Department of Pathology, University of Yamanashi, Chuo, Yamanashi, Japan; ${ }^{2}$ Division of Head and Neck Oncology, Cancer Institute Hospital of Japanese Foundation for Cancer Research, Tokyo, Japan; ${ }^{3}$ Division of Pathology, Cancer Institute of Japanese Foundation for Cancer Research, Tokyo, Japan; ${ }^{4}$ Department of Surgery, Ito Hospital, Tokyo, Japan and ${ }^{5}$ Department of Health Sciences, University of Yamanashi, Chuo, Yamanashi, Japan

Thyroid papillary carcinoma is the most common endocrine neoplasm and generally carries a favorable prognosis. However, a small subset of papillary carcinomas transforms into anaplastic carcinoma, an undifferentiated cancer with a dismal prognosis. Recent studies using next-generation sequencing revealed the genomic landscape of papillary carcinoma and anaplastic carcinoma. However, risk factors for anaplastic transformation in papillary carcinoma remain obscure. In the present study, we investigated molecular alterations of papillary carcinoma and anaplastic carcinoma components in 27 tumors in which anaplastic carcinoma coexisted with antecedent papillary carcinoma. We conducted direct sequencing for BRAF, TERT promoter and PIK3CA, and immunohistochemistry for p53, TTF-1 and subunits of the SWI/SNF complex (ARID1A, ARID1B, ATRX, SMARCA2, SMARCA4, SMARCB1, and PBRM1). BRAF ${ }^{V 600 E}$ and TERT promoter mutated at the rate of $90 \%$ and $95 \%$, respectively, and these mutational statuses were almost identical between the papillary carcinoma and anaplastic carcinoma components. PIK3CA mutation was positive in $33 \%$ of our samples with a heterogeneous mutation pattern of the papillary carcinoma and anaplastic carcinoma components. Aberrant expression of p53 and loss of TTF-1 were present in 63 and 59\%, respectively, and these two alterations were confined to the anaplastic carcinoma components. There was a loss of the SWI/SNF complex in a subset of the tumors with a heterogeneous pattern of the papillary carcinoma and anaplastic carcinoma components: SMARCA4 in 4\% and PBRM1 in 4\%. In a multivariate comparison between the antecedent papillary carcinoma components and control papillary carcinomas without anaplastic transformation, TERT promoter mutation was independently associated with anaplastic transformation. Collectively, papillary carcinoma-derived anaplastic carcinomas are characterized by BRAF and TERT promoter mutations, and these mutations occur prior to anaplastic transformation. Alterations of PIK3CA and the SWI/SNF complex are relatively rare and temporally heterogeneous. Of note, a papillary carcinoma harboring TERT promoter mutation is at higher risk for anaplastic transformation.

Modern Pathology (2017) 30, 1527-1537; doi:10.1038/modpathol.2017.75; published online 21 July 2017

Thyroid papillary carcinoma is the most common endocrine neoplasm, accounting for up to $90 \%$ of all thyroid cancers. ${ }^{1}$ The prognosis of patients with

Correspondence: Dr T Kondo, MD, PhD, Department of Pathology, University of Yamanashi, 1110 Shimokato, Chuo, Yamanashi 4093898, Japan.

E-mail: ktetsuo@yamanashi.ac.jp

Received 3 March 2017; revised 14 May 2017; accepted 15 May 2017; published online 21 July 2017 papillary carcinoma is generally favorable with a 10 -year overall survival rate of $>90 \%$. However, a small subset of papillary carcinoma tumors transform into a morphologically undifferentiated and clinically more aggressive cancer, anaplastic carcinoma. ${ }^{1-3}$ The prognosis of anaplastic carcinoma is usually dismal because of rapid tumor growth.

More recently, next-generation sequencing studies revealed the genomic landscapes of papillary carcinoma $^{4}$ and anaplastic carcinoma. ${ }^{5-9} B R A F^{V 600 E}$ 
mutation is the most frequent alteration in papillary carcinoma, ${ }^{4}$ and it remains the main mutation in anaplastic carcinoma. ${ }^{5}$ The TERT promoter mutation, which induces aberrant activation of telomerase, ${ }^{10,11}$ is identified in $\sim 10 \%$ of papillary carcinoma tumors and is closely associated with aggressive clinical behavior of papillary carcinoma. ${ }^{12-16}$ Notably, the frequency of TERT promoter mutation in anaplastic carcinoma is $40-70 \%,{ }^{12,13,15,17}$ which is significantly higher than in papillary carcinoma. ${ }^{9,12}$ In addition, mutations involving PIK $3 C A$ and the SWI/ SNF nucleosome remodeling complex are present in anaplastic carcinoma, ${ }^{5-9}$ whereas these mutations are rare or absent in papillary carcinoma. ${ }^{4,9,18}$ However, a detailed mechanism of the anaplastic transformation is still obscure. Particularly, it is unclear when these anaplastic carcinoma-related genetic alterations occur during the transformation from papillary carcinoma to anaplastic carcinoma. If the anaplastic carcinomarelated alterations occur prior to anaplastic transformation, then a papillary carcinoma tumor with these alterations should be considered as a high risk papillary carcinoma that is prone to undergo anaplastic transformation. To resolve this question, we investigated the respective papillary carcinoma and anaplastic carcinoma components in anaplastic carcinomas that contained antecedent papillary carcinoma.

The aim of this study is to determine the timing or sequence of the genetic alterations that occur during progression from papillary carcinoma to anaplastic carcinoma and to identify risk factors for anaplastic transformation. We examined molecular alterations in tumor samples from 27 cases of concomitant papillary carcinoma and anaplastic carcinoma. Subsequently, to identify risk factors for the transformation, we compared clinical and genetic features of the antecedent papillary carcinoma components in our samples with archived control papillary carcinomas that had not transformed, using univariate and multivariate models.

\section{Materials and methods}

Supplementary Figure S1 shows a schematic of the current study. This study was approved by the Institutional Review Board of University of Yamanashi (approval code: 1331), Ito Hospital (approval code: 150), and Japanese Institute for Cancer Research (approval code: 2016-1070).

\section{Patient Tissue Samples}

We enrolled 27 anaplastic carcinomas that contained antecedent papillary carcinoma components from the pathology archives of the University of Yamanashi Hospital, the Ito Hospital, and the Cancer Institute Hospital of Japanese Foundation for Cancer Research. To compile this case series, we examined 11480 papillary carcinomas and 228 anaplastic carcinomas in total. We performed additional pathological analyses on the formalin-fixed and paraffinembedded tissue blocks from these cases and obtained information pertaining to patients' ages and sex from the medical records under an appropriate de-identification. Two pathologists (NO and TK) evaluated histopathology and determined the histological variant of the antecedent papillary carcinoma components.

Our control group consisted of 92 archived papillary carcinomas with known statuses for $B R A F$ and TERT promoter. ${ }^{19}$ Moreover, from the open-access Cancer Genome Atlas Research Network (TCGA Research Network) study, ${ }^{4}$ we obtained 402 papillary carcinomas with their corresponding data on age, sex and BRAF and TERT statuses as another control group for further comparison.

\section{Mutation Analysis}

Using RecoverAll total nucleic acid isolation kit (Applied Biosystems, Tokyo, Japan), we extracted genomic DNA from formalin-fixed and paraffinembedded tissue. From anaplastic carcinomas with an antecedent papillary carcinoma component, each papillary carcinoma and anaplastic carcinoma component was carefully dissected as described previously. $^{20}$ After DNA extraction, we analyzed mutational statuses of BRAF (exon 15), TERT (promoter) and PIK3CA (exon 9 and exon 20) using standard direct sequencing. Supplementary Table S1 lists the primers used for the direct sequencing. In brief, $1 \mu \mathrm{l}$ of DNA in a final volume of $50 \mu \mathrm{l}$ with $10 \times$ PCR buffer, $200 \mu \mathrm{M}$ of each dNTP, $500 \mathrm{nM}$ of each primer and 1.25 units of the polymerase was amplified in the following thermal cycling condition: $15 \mathrm{~min}$ at $95^{\circ} \mathrm{C}$, followed by 40 cycles of $95^{\circ} \mathrm{C}$ for $30 \mathrm{~s}, 58^{\circ} \mathrm{C}$ for $30 \mathrm{~s}$ and $72^{\circ} \mathrm{C}$ for $30 \mathrm{~s}$, with a final extension at $72^{\circ} \mathrm{C}$ for $10 \mathrm{~min}$. After confirming specific amplification by electrophoresis, the PCR products were submitted to direct sequencing with each forward primer using BigDye Terminator v1.1 Cycle Sequencing Kit and ABI Prism 3130xl Genetic Analyzer (Applied Biosystems, Foster city, CA, USA).

\section{Immunohistochemistry}

We conducted standard, two-step, indirect immunohistochemistry to analyze the expressional status of p53, TTF-1 and subunits of the SWI/SNF complex (ARID1A, ARID1B, ATRX, SMARCA2, SMARCA4, SMARCB1, and PBRM1). Supplementary Table S2 provides detailed information on the antibodies, dilutions, and antigen retrieval. In brief, formalinfixed and paraffin-embedded tissue blocks were cut into $3-\mu \mathrm{m}$ sections, which were then de-paraffinized, rehydrated and blocked with $3 \%$ hydrogen peroxidase. After the antigen retrieval, each primary antibody was applied, and the slides were incubated at room temperature for $2 \mathrm{~h}$ (p53, TTF-1, ARID1A, 
Table 1 Clinical and genetic features of papillary carcinomas with or without anaplastic transformation (vs control papillary carcinomas)

\begin{tabular}{|c|c|c|c|c|c|c|c|c|}
\hline & \multirow{2}{*}{$\begin{array}{c}\text { Papillary carcinoma } \\
\text { components in anaplastic } \\
\text { carcinomas }(\mathrm{n}=27)\end{array}$} & \multirow{2}{*}{$\begin{array}{c}\text { Control papillary } \\
\text { carcinomas (overall) } \\
(\mathrm{n}=92)\end{array}$} & \multicolumn{3}{|c|}{ Univariate analysis } & \multicolumn{3}{|c|}{ Multivariate analysis } \\
\hline & & & $O R$ & $(95 \%$ CI) & P-value & OR & $(95 \%$ CI) & P-value \\
\hline \multicolumn{9}{|l|}{ Age (year) } \\
\hline Mean士s.d. & $68.1 \pm 8.2$ & $57.9 \pm 12.8$ & - & - & $<0.001$ & - & - & - \\
\hline Range & 51-84 & 9-96 & & & & & & \\
\hline$>60$ & $23(85 \%)$ & $38(41 \%)$ & 8.17 & $(2.61-25.5)$ & $<0.001$ & 1.30 & $(0.20-8.36)$ & 0.78 \\
\hline$\leq 60$ & $4(15 \%)$ & $54(59 \%)$ & 1 & (Reference) & & 1 & (Reference) & \\
\hline \multicolumn{9}{|l|}{ Sex } \\
\hline Female & $18(67 \%)$ & $57(62 \%)$ & 0.81 & $(0.33-2.01)$ & 0.82 & - & - & - \\
\hline Male & $9(33 \%)$ & $35(38 \%)$ & 1 & (Reference) & & & & \\
\hline \multicolumn{9}{|l|}{$B R A F^{V 600 E}$} \\
\hline Positive & $19(90 \%)$ & $77(84 \%)$ & 1.85 & $(0.39-8.79)$ & 0.34 & - & - & - \\
\hline Negative & $2(10 \%)$ & $15(16 \%)$ & 1 & (Reference) & & & & \\
\hline Not available & 6 & 0 & & & & & & \\
\hline \multicolumn{9}{|l|}{ TERT } \\
\hline $\mathrm{C} 228 \mathrm{~T}$ & $17(77 \%)$ & $8(9 \%)$ & 68.18 & $(13.97-332.79)$ & $<0.001^{\mathrm{a}}$ & 58.99 & $(9.25-376.37)$ & $<0.001^{\mathrm{a}}$ \\
\hline $\mathrm{C} 250 \mathrm{~T}$ & $3(14 \%)$ & $3(4 \%)$ & & & & & & \\
\hline Wild type & $2(9 \%)$ & $75(87 \%)$ & 1 & (Reference) & & 1 & (Reference) & \\
\hline Not available & 5 & 6 & & & & & & \\
\hline
\end{tabular}

Abbreviations: CI, confidence interval; OR, odds ratio.

a Mutant (C228T and C250T) vs wild type.

ARID1B, SMARCA2, SMARCA4, SMARCB1, and PBRM1) or at $4^{\circ} \mathrm{C}$ overnight (ATRX). After incubation with a secondary antibody (Histofine Simple Stain MAX-PO; Nichirei Bioscience, Tokyo, Japan) at room temperature for $30 \mathrm{~min}$, the sections were developed with 3,3'-diaminobenzidine and counterstained with hematoxylin.

We considered immunohistochemistry for p53 as positive if more than $70 \%$ of tumor cells exhibited intense signal. If $<10 \%$ of the tumor cells in one high-power field lacked nuclear expression, we determined it to be an aberrant loss of TTF-1 and SWI/SNF complex. We confirmed the positivity in normal follicular epithelial cells and stromal cells as internal positive controls for TTF-1 and SWI/SNF complex, respectively.

\section{Statistics}

Chi-square test and the Fisher's exact test were used for ordinal or nominal categorical variables and discrete numerical variables. Student's $t$-test and Mann-Whitney test were used for continuous numerical data after the Shapiro-Wilk test for normality. We used univariate and multivariate analyses in logistic regression to calculate odd ratios and $95 \%$ confidence intervals. For the multivariate analysis, we included explanatory variables with a $P$-value of $<0.25$ in the univariate analyses. All statistical calculations were performed with the SPSS Statistics version 22 (IBM Japan, Tokyo, Japan). All reported $P$-values were two-sided, and $P$-value of $<0.05$ was considered statistically significant.

\section{Results}

\section{Clinicopathological Features}

Table 1 summarizes the clinical data of 27 anaplastic carcinomas with antecedent papillary carcinoma components. The patients were elderly adults with a mean age at surgery of 68.1 years (range: 51-84). Females accounted for $67 \%(18 / 27)$ of the patients. The histology of the papillary carcinoma component was classical in $81 \%(22 / 27)$ of the tumors, tall cell variant in $7 \%(2 / 27)$, invasive follicular variant in $7 \%(2 / 27)$, and solid variant in $4 \%(1 / 27)$.

\section{BRAF Mutation}

Figure 1a summarizes the mutational profile of each papillary carcinoma and anaplastic carcinoma component in the 27 tumors; Genetic status of $B R A F$ was informative in 21 tumors, and $B R A F$ mutation was positive in $90 \%(19 / 21)$ of the samples. All the $B R A F$ mutations were $B R A F^{V 600 E}$. Paired $B R A F$ status of papillary carcinoma and anaplastic carcinoma components was available in 18 tumors, and all 18 tumors shared an identical $B R A F$ genotype between the papillary carcinoma and anaplastic carcinoma components. Figure 2, shows a representative histopathology and $B R A F$ status of case \#9.

\section{TERT Promoter Mutation}

Genetic status of the TERT promoter was informative in 22 out of 27 tumors. TERT promoter mutation was 
a
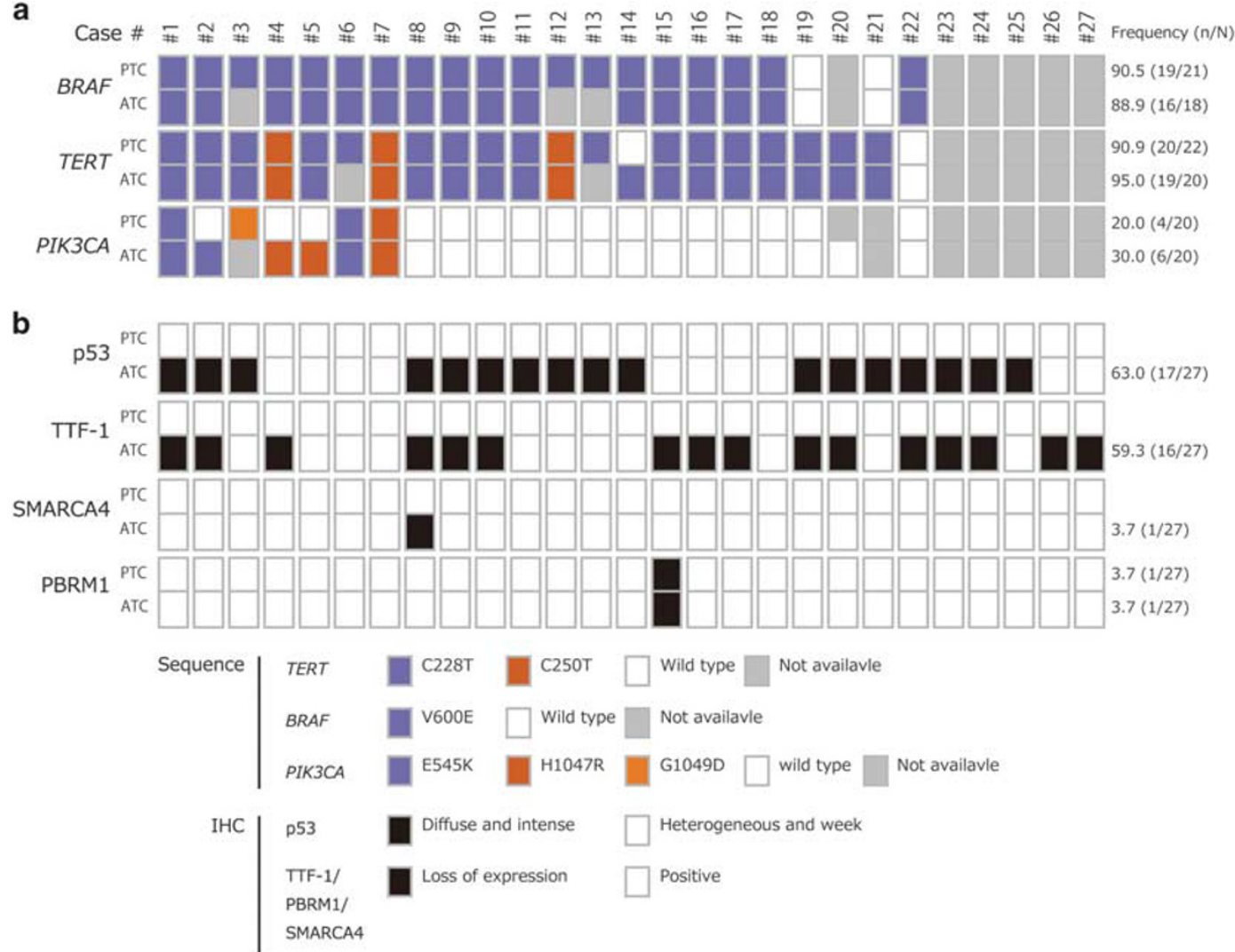

Figure 1 Summary of (a) genetic and (b) immunohistochemical analyses. The upper and lower boxes indicate the papillary carcinoma (PTC) and anaplastic carcinoma (ATC) component, respectively.

positive in $95 \%(21 / 22)$ of the tumors (Figure 1a), and of these TERT-mutated tumors, chromosome 5: $1,295,228 \mathrm{C}>\mathrm{T}(\mathrm{C} 228 \mathrm{~T})$ accounted for $86 \%(18 / 21)$, whereas chromosome 5: 1,295,250 C > T (C250T) was identified in $14 \%(3 / 21)$. The TERT-mutated tumors had $B R A F^{V 600 E}$ mutation in $90 \%(19 / 21)$ of the samples. Paired TERT promoter status of the papillary carcinoma and anaplastic carcinoma components was available in 20 tumors, and an identical TERT promoter genotype was evident in 95\% (19/20). One tumor had a discordant TERT mutational status between the papillary carcinoma and anaplastic carcinoma components: TERT promoter C228T mutation was positive in the anaplastic carcinoma component but not in the antecedent papillary carcinoma component (Figure 1a, \#14). Figure 2, from case \#9, shows a TERT promoter mutation.

\section{PIK3CA Mutation}

As shown in Figure 1a, we could determine the genetic status of PIK3CA in 21 out of 27 tumors. There were PIK3CA mutations in 33\% (7/21): three E545K (exon 9), three H1047R (exon 20) and one G1049D (exon 20). The mutational patterns between papillary carcinoma and anaplastic carcinoma components were heterogeneous. Among the six PIK3CA-mutated tumors in which paired papillary carcinoma and anaplastic carcinoma data were available, $50 \%$ (3/6) exhibited a shared PIK3CA mutation between the papillary carcinoma and anaplastic carcinoma components, whereas the remaining 50\% (3/6) harbored the mutation exclusively in the anaplastic carcinoma component. The all (6/6) PIK3CA-muated tumors were positive for both $B R A F^{V 600 E}$ and TERT promoter mutations. Figure 3 shows a representative tumor (case \#2), with a discordant PIK3CA genotype between papillary carcinoma and anaplastic carcinoma components.

\section{Immunohistochemistry for TTF-1 and p53}

Figure 1b shows the successful immunohistochemistry for all 27 tumors. In accordance with previous studies, ${ }^{21,22}$ loss of TTF-1 expression was evident in $59 \%(16 / 27)$ of our samples, and this aberrant expression was confined to the anaplastic carcinoma components (Figure 2). We identified diffuse and intense expression of p53 protein in 63\% (17/27), and this aberrant p53 expression also was evident only in the anaplastic carcinoma components, whereas all papillary carcinoma components showed heterogeneous and weak p53 expression (Figure 2). 

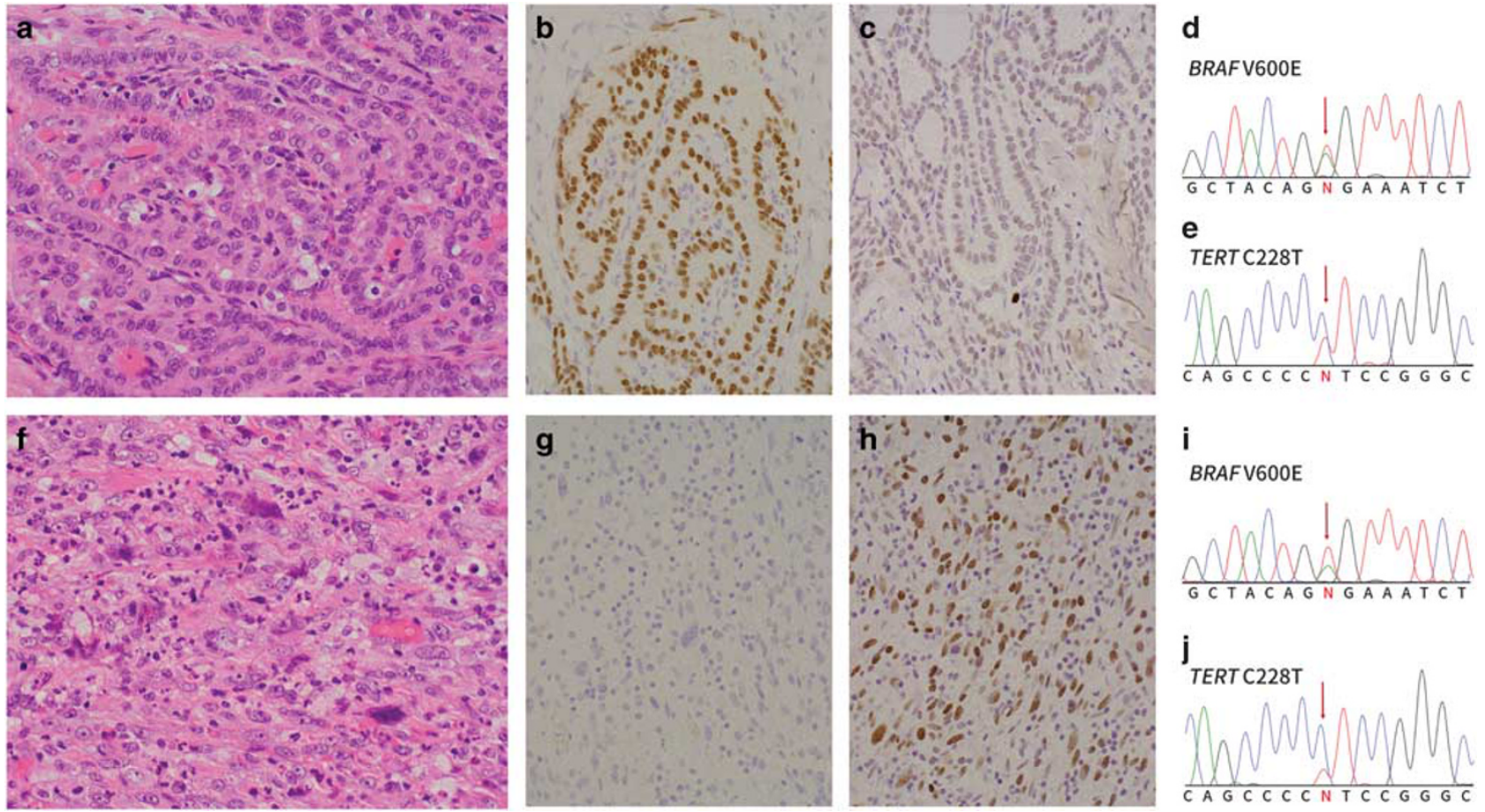

Figure 2 Representative histopathology and molecular alterations of (a-e) papillary carcinoma and (f-h) anaplastic carcinoma components in case \#9. TTF-1 is diffusely positive in the (b) papillary carcinoma component, whereas it is totally negative in the (g) anaplastic carcinoma component. Conversely, p53 is weak and heterogeneous in the (c) papillary carcinoma component but diffusely positive in the (h) anaplastic carcinoma component. Both papillary carcinoma and anaplastic carcinoma components harbor the (d and i) $B R A F^{\mathrm{V} 600 \mathrm{E}}$ and (e and j) TERT promoter mutation 1,295,228 C > T (C228T).

BRAFV600E

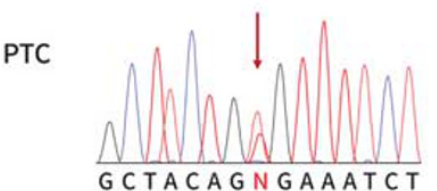

d

BRAFV600E

ATC

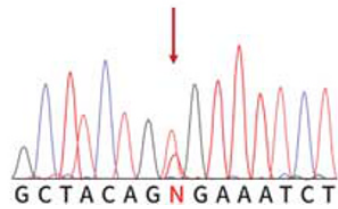

b

TERT C228T

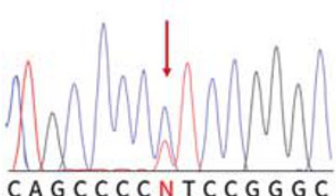

e

TERT C228T

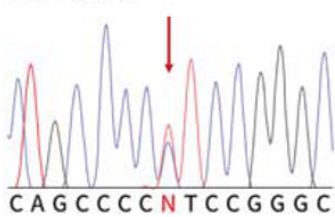

PIK3CAWT

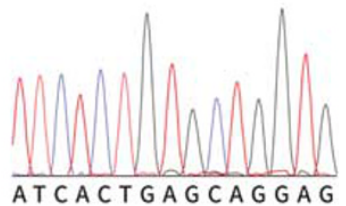

f

PIK3CA E545K

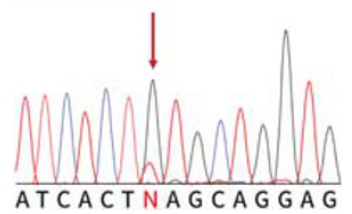

Figure 3 Direct sequencing of (a-c) papillary carcinoma and (d-f) anaplastic carcinoma components of case \#2. The (a and d) $B R A F^{\mathrm{V} 600 \mathrm{E}}$ and (b and e) TERT promoter mutation chromosome 5: 1,295,228 C > T (C228T) are shared between papillary carcinoma and anaplastic carcinoma components. PIK3CA E545K is evident in the (f) anaplastic carcinoma but not in the (e) papillary carcinoma component.

\section{Immunohistochemistry for the SWI/SNF Complex}

As shown in Figure 1b, altered expression of the SWI/SNF complex was immunohistochemically identified in $7 \%(2 / 27)$ of the samples. Case \#8 had aberrant loss of expression of SMARCA4, and case
\#15 had aberrant loss of expression of PBRM1. Loss of SMARCA4 expression in case \#8 was limited to the central area of the anaplastic carcinoma component, whereas the papillary carcinoma component and periphery of the anaplastic carcinoma component retained the expression (Figure 4). In contrast, 

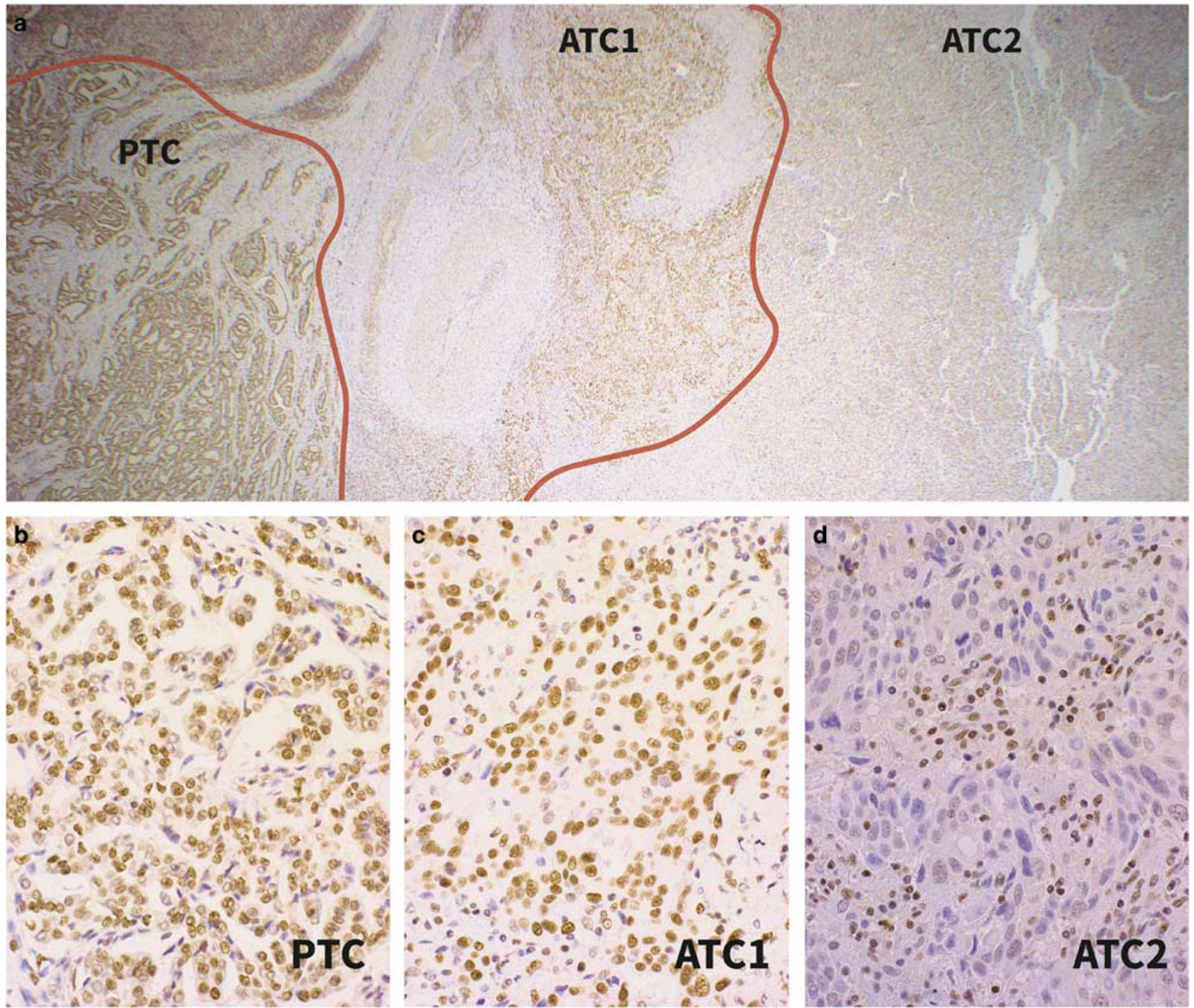

Figure 4 Expression of SMARCA4 in case \#8. Although nuclear expression SMARCA4 is retained in the (a and b: PTC) antecedent papillary carcinoma and the (a and $\mathbf{c}$ : ATC1) peripheral area of the anaplastic carcinoma component, the (a and d: ATC2) central area of anaplastic carcinoma lacks the expression.

PBRM1 expression of case \#15 was entirely lost in both the papillary carcinoma and anaplastic carcinoma components (Figure 5).

\section{Risk Factors for Anaplastic Transformation}

To explore risk factors for anaplastic transformation of papillary carcinoma, we compared clinical and genetic features between the 27 antecedent papillary carcinomas that underwent anaplastic transformation and the 92 archived control papillary carcinomas without anaplastic transformation. Table 1 summarizes the results of the univariate and multivariate analyses on the relation of patients' ages, sex, and $B R A F^{V 600 E}$ and TERT promoter mutations with anaplastic transformation. In the univariate analyses, anaplastic transformation was significantly associ- ated with an age $>60$ years (85 vs $41 \% ; P<0.001$ ) and the presence of the TERT promoter mutation (91 vs $13 \% ; P<0.001)$. In the multivariate analysis looking at both age (older than 60 years) and TERT promoter mutation, the TERT promoter mutation (odds ratio, 58.99; 95\% confidence interval, 9.25376.37) was associated with anaplastic transformation but age $(P=0.78)$ was not.

Subsequently, we excluded micro-papillary carcinomas (papillary carcinomas measuring $1.0 \mathrm{~cm}$ or smaller) from the control papillary carcinomas. We based this choice on a hypothesis that micropapillary carcinomas never transform into anaplastic carcinomas, and maintaining micro-papillary carcinomas in the control group might then bias the risk assessment for anaplastic carcinoma. Table 2 indicates the results of the univariate and multivariate analyses after removing the micro-papillary 

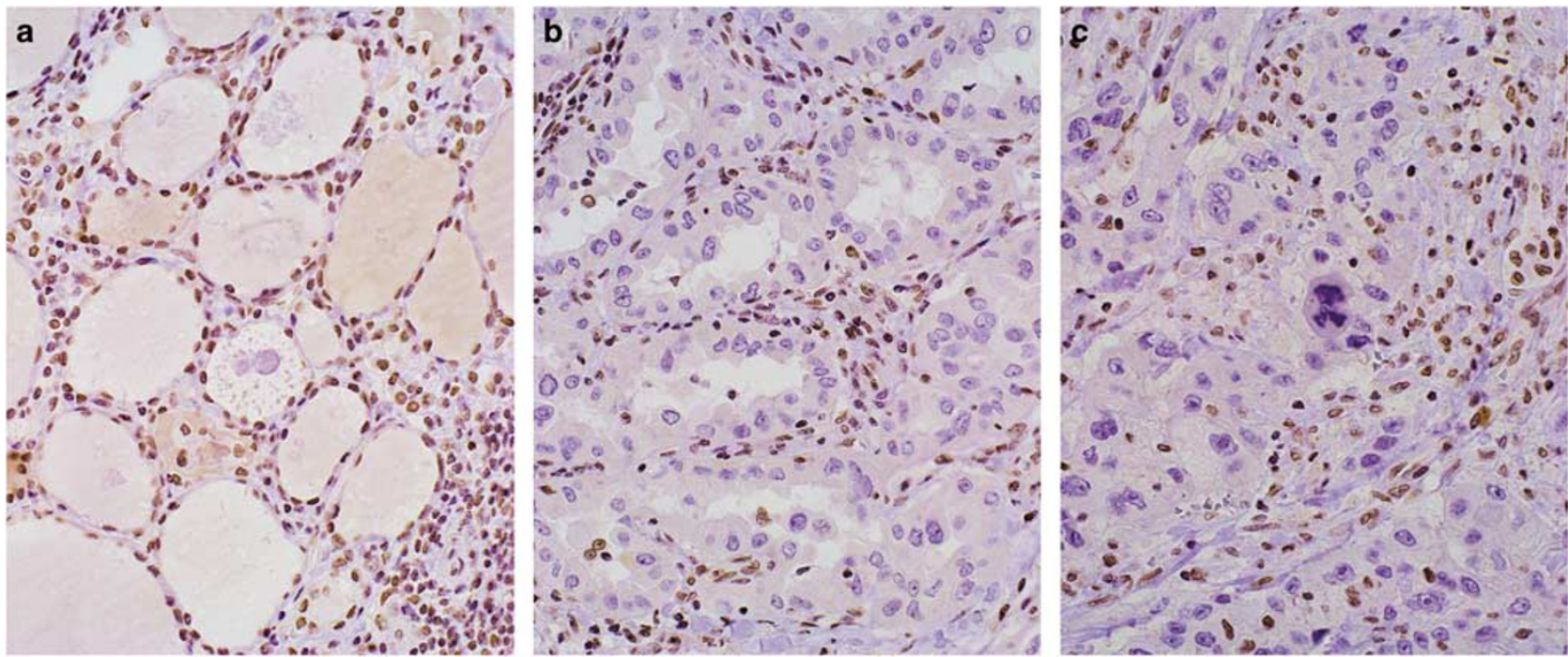

Figure 5 Expression of PBRM1 in (a) normal, (b) papillary carcinoma and (c) anaplastic carcinoma components of case \#15. Although (a) normal follicular epithelial cells are positive for PBRM1, both (b) papillary carcinoma and (c) anaplastic carcinoma components lack the nuclear expression of PBRM1. Note the positivity in endothelial cells and infiltrating lymphocytes as an internal positive control.

carcinomas. As in the prior analysis that included overall papillary carcinomas, we found that being older than 60 years (85 vs $37 \% ; P<0.001)$ and having the TERT promoter mutation (91 vs $14 \%$; $P<0.001)$ were both associated with anaplastic transformation in the univariate analyses. However, in the multivariate analysis, only the presence of the TERT promoter mutation (odds ratio, 42.34; 95\% confidence interval, 6.51-275.41) remained a significant risk factor.

To consolidate the evidence that TERT promoter mutation in papillary carcinoma is an independent risk factor for anaplastic transformation, we compared our results with open-access data of 402 papillary carcinomas in the TCGA study, ${ }^{4}$ which is the most comprehensive study of papillary carcinoma so far, using univariate and multivariate analyses (Table 3). In the univariate analyses, anaplastic transformation was significantly associated with a patient age $>60$ years (85 vs $21 \% ; P<0.001$ ), $B R A F^{V 600 E}$ mutation (90 Vs $\left.58 \% ; P=0.003\right)$ and TERT promoter mutation (91 vs $9 \% ; P<0.001$ ). The multivariate analysis, which included age and both $B R A F^{V 600 E}$ and TERT promoter mutations, revealed that patient age greater than 60 years (odds ratio, 4.87; 95\% confidence interval, 1.17-20.37) and TERT promoter mutation (odds ratio, 39.48; $95 \%$ confidential interval, 7.93-196.56) remained independently associated with anaplastic transformation, whereas BRAF V600E $(P=0.066)$ was no longer a significant factor.

\section{Discussion}

Anaplastic carcinoma is the most aggressive and lethal of all human cancers, ${ }^{1}$ and a subset of them originates from papillary carcinoma. ${ }^{1,3,20,23}$ Molecular studies provide evidence for this progression theory. A recent next-generation sequencing study reported that anaplastic carcinoma harbors a higher number of mutations than papillary carcinoma,${ }^{5}$ suggesting gradual accumulation of somatic mutations during progression from papillary carcinoma to anaplastic carcinoma. Moreover, Hunt et $a l^{24}$ demonstrated that the majority of tumors with concomitant papillary carcinoma and anaplastic carcinoma components share the tumor suppressor mutations. In oncogenic alterations, BRAF V600E is the most common point mutation in papillary carcinoma, ${ }^{4,25-27}$ and it is present in up to $50 \%$ of anaplastic carcinomas. ${ }^{5-9}$ Other studies also demonstrate that in anaplastic carcinomas that contain papillary carcinoma components, $B R A F^{V 600 E}$ is found in both the papillary carcinoma and anaplastic carcinoma components, ${ }^{3,20,28}$ suggesting early acquisition of $B R A F^{V 600 E}$ and the clonal evolution of anaplastic carcinoma from papillary carcinoma. The present study confirms the multistep progression theory of papillary carcinoma and provides further insights into the order of mutations during this progression and the risk factors for anaplastic transformation.

This is the first study that demonstrated an association between TERT promoter mutation and anaplastic transformation. As the TERT promoter mutation is closely correlated with older patient's age, ${ }^{14,19,29,30}$ we needed to perform a multivariate analysis that included both age and TERT mutation to evaluate the actual impact of TERT promoter mutation on anaplastic transformation. Consequently, the multivariate logistic regression analysis demonstrated that TERT promoter mutation is an independent risk factor for anaplastic 
transformation. Furthermore, the independent association of patient age found in the TCGA study was limited as shown by its lower estimated odds ratio compared with the TERT promoter mutation. This finding implies that the TERT promoter mutation may be a more powerful indicator of transformation

Table 2 Clinical and genetic features of papillary carcinomas with or without anaplastic transformation (vs control papillary carcinomas larger than $1.0 \mathrm{~cm}$ )

\begin{tabular}{|c|c|c|c|c|c|c|c|c|}
\hline & \multirow{2}{*}{$\begin{array}{l}\text { Papillary carcinoma } \\
\text { components in anaplastic } \\
\text { carcinomas }(\mathrm{n}=27)\end{array}$} & \multirow{2}{*}{$\begin{array}{c}\text { Control papillary } \\
\text { carcinomas }(>1.0 \mathrm{~cm}) \\
(\mathrm{n}=46)\end{array}$} & \multicolumn{3}{|c|}{ Univariate analysis } & \multicolumn{3}{|c|}{ Multivariate analysis } \\
\hline & & & $O R$ & $(95 \%$ CI) & P-value & OR & $(95 \%$ CI) & $\mathrm{P}$-value \\
\hline \multicolumn{9}{|l|}{ Age (year) } \\
\hline Mean土s.d. & $68.1 \pm 8.2$ & $57.5 \pm 14.7$ & - & - & $<0.001$ & - & - & - \\
\hline Range & $51-84$ & $9-96$ & & & & & & \\
\hline$>60$ & $23(85 \%)$ & $17(37 \%)$ & 9.81 & $(2.91-33.19)$ & $<0.001$ & 2.19 & $(0.33-14.77)$ & 0.42 \\
\hline$\leq 60$ & $4(15 \%)$ & $29(63 \%)$ & & & & 1 & (Reference) & \\
\hline \multicolumn{9}{|l|}{ Sex } \\
\hline Female & 18 (67\%) & $26(57 \%)$ & 0.65 & $(0.24-1.75)$ & 0.46 & - & - & - \\
\hline Male & $9(33 \%)$ & $20(43 \%)$ & 1 & (Reference) & & & & \\
\hline \multicolumn{9}{|l|}{ BRAF V600E } \\
\hline Positive & $19(90 \%)$ & 38 (83\%) & 2.00 & $(0.39-10.36)$ & 0.33 & - & - & - \\
\hline Negative & $2(10 \%)$ & $8(17 \%)$ & 1 & (Reference) & & & & \\
\hline Not available & 6 & 0 & & & & & & \\
\hline \multicolumn{9}{|l|}{ TERT } \\
\hline $\mathrm{C} 228 \mathrm{~T}$ & $17(77 \%)$ & $4(9 \%)$ & 63.33 & (11.69-343.03) & $<0.001^{\mathrm{a}}$ & 42.34 & $(6.51-275.41)$ & $<0.001^{\mathrm{a}}$ \\
\hline $\mathrm{C} 250 \mathrm{~T}$ & $3(14 \%)$ & $2(5 \%)$ & & & & & & \\
\hline Wild type & $2(9 \%)$ & $38(86 \%)$ & 1 & (Reference) & & 1 & (Reference) & \\
\hline Not available & 5 & 2 & & & & & & \\
\hline
\end{tabular}

Abbreviations: CI, confidence interval; OR, odds ratio.

${ }^{\mathrm{a}}$ Mutant (C228T and C250T) vs wild type.

Table 3 Clinical and genetic features of papillary carcinomas with or without anaplastic transformation (vs control papillary carcinomas in the TCGA study)

\begin{tabular}{|c|c|c|c|c|c|c|c|c|}
\hline & \multirow{2}{*}{$\begin{array}{l}\text { Papillary carcinoma } \\
\text { components in anaplsatic } \\
\text { carcinomas }(\mathrm{n}=27)\end{array}$} & \multirow{2}{*}{$\begin{array}{c}\text { Control papillary } \\
\text { carcinomas (TCGA) } \\
(\mathrm{n}=402)\end{array}$} & \multicolumn{3}{|c|}{ Univariate analysis } & \multicolumn{3}{|c|}{ Multivariate analysis } \\
\hline & & & OR & $(95 \%$ CI) & $\mathrm{P}$-value & OR & $(95 \% C I)$ & $\mathrm{P}$-value \\
\hline \multicolumn{9}{|l|}{ Age (year) } \\
\hline Mean \pm s.d. & $68.1 \pm 8.2$ & $46.8 \pm 15.3$ & - & - & $<0.001$ & - & - & - \\
\hline Range & $51-84$ & 17-89 & & & & & & \\
\hline$>60$ & $23(85 \%)$ & $81(21 \%)$ & 21.01 & (7.07-62.49) & $<0.001$ & 4.87 & (1.17-20.37) & 0.030 \\
\hline$\leq 60$ & $4(15 \%)$ & $296(79 \%)$ & 1 & (Reference) & & 1 & (Reference) & \\
\hline Not available & & 25 & & & & & & \\
\hline \multicolumn{9}{|l|}{ Sex } \\
\hline Female & $18(67 \%)$ & $281(75 \%)$ & 1.46 & $(0.64-3.37)$ & 0.37 & - & - & - \\
\hline Male & $9(33 \%)$ & $96(25 \%)$ & 1 & (Reference) & & & & \\
\hline Not available & & 25 & & & & & & \\
\hline \multicolumn{9}{|l|}{$B R A F^{V 600 E}$} \\
\hline Positive & $19(90 \%)$ & $235(58 \%)$ & 6.75 & $(1.55-29.38)$ & 0.003 & 4.57 & $(0.90-23.05)$ & 0.066 \\
\hline Negative & $2(10 \%)$ & $167(42 \%)$ & 1 & (Reference) & & 1 & (Reference) & \\
\hline Not available & 6 & 0 & & & & & & \\
\hline \multicolumn{9}{|l|}{ TERT } \\
\hline $\mathrm{C} 228 \mathrm{~T}$ & $17(77 \%)$ & $27(7 \%)$ & 96.67 & $(21.71-430.42)$ & $<0.001^{\mathrm{a}}$ & 39.48 & (7.93-196.56) & $<0.001^{\mathrm{a}}$ \\
\hline $\mathrm{C} 250 \mathrm{~T}$ & $3(14 \%)$ & $8(2 \%)$ & & & & & & \\
\hline C228A & 0 & 1 & & & & & & \\
\hline Wild type & $2(9 \%)$ & 348 (91\%) & 1 & (Reference) & & 1 & (Reference) & \\
\hline Not available & 5 & 18 & & & & & & \\
\hline
\end{tabular}

Abbreviations: CI, confidence interval; OR, odds ratio; TCGA, The Cancer Genome Atras.

${ }^{\mathrm{a}}$ Mutant (C228T, C250T and C228A) vs wild type. 
than patient age, although older patient age has been recognized as a risk factor for anaplastic carcinoma. ${ }^{1}$

The higher risk for transformation of TERTmutated papillary carcinomas is consistent with previous molecular studies on the biology of TERTmutated papillary carcinomas. First, akin to anaplastic carcinoma, TERT-mutated papillary carcinomas are functionally less differentiated. The TCGA study demonstrated that TERT-mutated papillary carcinomas have lower expression levels of thyroid metabolism and function genes than TERT wild-type papillary carcinomas; ${ }^{4}$ anaplastic carcinoma is characterized by functional dedifferentiation from thyroid follicular epithelial cells. ${ }^{5}$ For example, TTF-1/NKX2.1 gene, which codes an essential transcriptional factor for the thyroid, is epigenetically down regulated in anaplastic carcinoma cells. ${ }^{22}$ Second, telomere length in TERT-mutated papillary carcinoma is shorter than in TERT wild-type papillary carcinoma. ${ }^{30}$ As a shorter telomere is associated with aging of tumor cells, the TERT promoter mutation is a late-stage event in the progression of papillary carcinoma. Furthermore, a shorter telomere is associated with chromosomal instability, and that is an important feature of anaplastic carcinoma. ${ }^{31}$ Collectively, papillary carcinoma with TERT promoter mutation represents the later phase of progression with a less-differentiated status and chromosomal instability, and it is prone to undergo anaplastic transformation.

In the present study, we did not find an independent association between $B R A F$ mutation and anaplastic transformation, whereas, in previous studies, $B R A F$ mutation was found to be a prognostic factor of papillary carcinoma, ${ }^{32,33}$ and papillary carcinoma with dual-mutation of $B R A F$ and TERT promoter was demonstrated as having the worst prognosis. ${ }^{14,34}$ However, even in the comparison with the TCGA study that included less $B R A F$-mutated papillary carcinomas than our control papillary carcinomas, $B R A F$ mutation did not remain an independent risk factor. This discrepancy may be due to our samples because nearly all of the TERT-mutated tumors in our study were also $B R A F$-mutated. A small number of TERT-mutated and BRAF-wild type tumors may have resulted in the absence of independent association.

The gain of function PIK3CA mutation was positive in one third of our series of anaplastic carcinomas, and there was a heterogeneous mutation pattern between the papillary carcinoma and anaplastic carcinoma components. According to recent studies using next-generation-sequencing, mutations affecting the PI3K-AKT-mTOR pathway were present in up to $39 \%$ of anaplastic carcinomas, and most of the mutations were of PIK $3 C A .^{5-9}$ In contrast, PIK3CA mutation is generally rare $(<3 \%)$ in typical papillary carcinomas ${ }^{4,18}$ but frequent (31\%) in papillary carcinomas with distant metastases. ${ }^{35}$ In our study, antecedent papillary carcinoma components in anaplastic carcinomas harbored PIK3CA mutation at a higher frequency $(20 \%)$ than that found in typical papillary carcinomas. Taken together, we can conclude that PIK3CA mutation is a late-stage event in the progression of papillary carcinoma to anaplastic carcinoma: it is almost non-existent or sub-clonal in typical papillary carcinomas but more frequent in aggressive and/or advanced papillary carcinomas. Our finding is consistent with a previous study by Charles et al. ${ }^{36}$ in which they developed a mouse papillary carcinoma model that had a conditional PIK3CA mutation. By activating mutations of $B R A F$ and PIK3CA, they showed that the mutations cooperate to promote carcinogenesis of anaplastic carcinoma. Nonetheless, unlike the BRAF and TERT mutations, PIK3CA mutation is an event that is temporally heterogeneous before and after the transformation. Actually, half of the PIK3CAmutated anaplastic carcinomas in the current study lacked the mutation in their antecedent papillary carcinoma components. Therefore, the presence of the PIK3CA mutation may be less useful than the TERT promoter mutation for determining the risk assessment for anaplastic transformation.

We found a loss of protein expression of the SWI/ SNF complex in a subset of anaplastic carcinomas with a papillary carcinoma component. Moreover, similar to PIK3CA mutation, these alterations are acquired with heterogeneous timing. Alterations involving the SWI/SNF complex have been identified in various human cancers. ${ }^{37}$ In thyroid cancer, a next-generation-sequencing study by Landa et al showed that $36 \%$ of anaplastic carcinomas harbor mutations of the SWI/SNF complex including ARID1A, ARID1B, ARID2, ARID5B, SMARCB1, $P B R M 1$, and $A T R X,{ }^{5}$ whereas these mutations are rare in papillary carcinoma. ${ }^{4}$ As most of the SWI/ SNF alterations are somatic missense or truncating, 5 these alterations can be detected as loss of protein expression with immunohistochemistry. ${ }^{37,38}$ Indeed, Wiegand et al $^{39}$ examined expression of ARID1A in a series of various cancers including thyroid cancer and reported loss of ARID1A protein in 14\% of anaplastic carcinomas. In the present study, we evaluated the expression of multiple SWI/SNF subunits by paneled immunohistochemistry of ARID1A, ARID1B, ATRX, SMARCA2, SMARCA4, SMARCB1, and PBRM1. The frequency of abnormal SWI/SNF complex in our study was lower than that in previous studies. ${ }^{5,39}$ However, one tumor exhibited loss of SMRACA4 in the anaplastic carcinoma component but not in the papillary carcinoma component, and one tumor had loss of PBRM1 in both papillary carcinoma and anaplastic carcinoma components. This finding indicates that mutation of the SWI/SNF complex is a later event in the progression of thyroid cancer having a heterogeneous timing around the transformation. Recent studies have shown that SWI/SNF-mutant cancers depend on residual unaffected SWI/SNF complexes for their aberrant growth, revealing synthetic lethal interactions that could be exploited for therapeutic 
purposes. ${ }^{40}$ Therefore, SWI/SNF mutations could be a novel therapeutic target in anaplastic carcinoma.

Unlike the above-described molecular alterations, aberrant p53 expression and loss of TTF-1 expression were present exclusively in anaplastic carcinoma components, suggesting that these alterations are directly associated with anaplastic transformation. TP53 mutation, which may be detectable as diffuse and intense positivity in immunohistochemistry for p53 protein, is highly prevalent in anaplastic carcinomas but rare in overall papillary carcinomas. ${ }^{5-9,41}$ Furthermore, mutant p53 has been shown to drive progression to anaplastic carcinoma in a $B R A F$-mutant mouse model of papillary carcinoma. $^{42}$ Conversely, loss of TTF-1, a master regulator of thyroid differentiation, is a hallmark of anaplastic carcinoma, whereas differentiated thyroid carcinomas including papillary carcinoma express TTF-1. ${ }^{21}$ The loss of TTF-1 expression is mainly owing to the epigenetic silencing via promoter methylation and histone modification in anaplastic carcinoma. $^{22}$ In the present study, even papillary carcinoma components within anaplastic carcinomas, which may be regarded as a terminal phase in the progression of papillary carcinoma, had not yet exhibited aberrant p53 expression or loss of TTF-1. Hence, our study further evidenced the theory that TP53 mutation and loss of TTF-1 are key distinguishing events between differentiated thyroid carcinoma and anaplastic carcinoma.

Despite our novel findings, the present study has several limitations. First, as our study focused on molecular alterations during the transformation from papillary carcinoma to anaplastic carcinoma, we investigated only anaplastic carcinomas that contained a papillary carcinoma component. Etiologically, anaplastic carcinoma is a heterogeneous cancer with various precursors. ${ }^{1,2}$ For example, follicular thyroid carcinoma also can be a precursor of anaplastic carcinoma. Moreover, some anaplastic carcinomas may arise de novo. Our study did not include these non-papillary carcinoma-originated anaplastic carcinomas. Second, all of the anaplastic carcinomas in the current study were surgically resected cases. Because of its rapid growth, anaplastic carcinoma is generally unresectable and diagnosed by biopsy or aspiration cytology. Therefore, it should be noted that anaplastic carcinomas in the present study were selected and do not necessarily represent overall anaplastic carcinomas. Finally, this was a retrospective study. A long-term prospective study of TERT-mutated papillary carcinomas would help to consolidate the role of the TERT promoter mutation in anaplastic transformation.

In conclusion, we analyzed molecular alterations of concomitant papillary carcinoma and anaplastic carcinoma. More than $90 \%$ of our tumor samples harbored $B R A F^{V 600 E}$ and TERT promoter mutations, and these mutations were seen in both the papillary carcinoma and anaplastic carcinoma components. Alterations of PIK3CA and the SWI/SNF chromatin- remodeling complex were relatively rare with a heterogeneous pattern of acquisition. Abnormal expression of p53 and TTF-1 were frequent but identified exclusively in anaplastic carcinoma components. In the multivariate analysis of papillary carcinomas without anaplastic transformation, TERT promoter mutation was independently associated with anaplastic transformation. Consequently, TERT-mutated papillary carcinoma is not only indicative of the clinical aggressiveness of the papillary carcinoma but also the higher risk for progression to anaplastic carcinoma.

\section{Acknowledgments}

This study was supported by JSPS KAKENHI (Grant Number 15K19050). We also thank Ms Wakaba Iha, Ms Miyuki Itoh, Ms Mikiko Yoda and Mr Yoshihito Koshimizu for technical support, and M Kayoko Kono for executive assistance.

\section{Disclosure/conflict of interest}

The authors declare no conflict of interest.

\section{References}

1 Cabanillas ME, McFadden DG, Durante C. Thyroid cancer. Lancet 2016;388:2783-2795.

2 Patel KN, Shaha AR. Poorly differentiated and anaplastic thyroid cancer. Cancer Control 2006;13:119-128.

3 Quiros RM, Ding HG, Gattuso P, et al. Evidence that one subset of anaplastic thyroid carcinomas are derived from papillary carcinomas due to BRAF and p53 mutations. Cancer 2005;103:2261-2268.

4 The Cancer Genome Atlas Reseach Network. Integrated genomic characterization of papillary thyroid carcinoma. Cell 2014;159:676-690.

5 Landa I, Ibrahimpasic T, Boucai L, et al. Genomic and transcriptomic hallmarks of poorly differentiated and anaplastic thyroid cancers. J Clin Invest 2016;126: 1052-1066.

6 Sykorova V, Dvorakova S, Vcelak J, et al. Search for new genetic biomarkers in poorly differentiated and anaplastic thyroid carcinomas using next generation sequencing. Anticancer Res 2015;35:2029-2036.

7 Kunstman JW, Juhlin CC, Goh G, et al. Characterization of the mutational landscape of anaplastic thyroid cancer via whole-exome sequencing. Hum Mol Genet 2015;24:2318-2329.

8 Jeon MJ, Chun S-M, Kim D, et al. Genomic alterations of anaplastic thyroid carcinoma detected by targeted massive parallel sequencing in a BRAF V600E mutation-prevalent area. Thyroid 2016;26:683-690.

$9 \mathrm{Xu} \mathrm{B}$, Ghossein R. Genomic landscape of poorly differentiated and anaplastic thyroid carcinoma. Endocr Pathol 2016;27:205-212.

10 Huang FW, Hodis E, Xu MJ, et al. Highly recurrent TERT promoter mutations in human melanoma. Science 2013;339:957-959. 
11 Horn S, Figl A, Rachakonda PS, et al. TERT promoter mutations in familial and sporadic melanoma. Science 2013;339:959-961.

12 Liu R, Xing M. TERT promoter mutations in thyroid cancer. Endocr Relat Cancer 2016;23:R143-R155.

13 Liu X, Bishop J, Shan Y, et al. Highly prevalent TERT promoter mutations in aggressive thyroid cancers. Endocr Relat Cancer 2013;20:603-610.

14 Xing M, Liu R, Liu X, et al. BRAF V600E and TERT promoter mutations cooperatively identify the most aggressive papillary thyroid cancer with highest recurrence. J Clin Oncol 2014;32:2718-2726.

15 Landa I, Ganly I, Chan Ta, et al. Frequent somatic TERT promoter mutations in thyroid cancer: higher prevalence in advanced forms of the disease. J Clin Endocrinol Metab 2013;98:E1562-E1566.

16 Vinagre J, Almeida A, Pópulo $\mathrm{H}$, et al. Frequency of TERT promoter mutations in human cancers. Nat Commun 2013;4:2185.

17 Shi X, Liu R, Qu S, et al. Association of TERT promoter mutation 1,295,228 C > T with BRAF V600E mutation, older patient age, and distant metastasis in anaplastic thyroid cancer. J Clin Endocrinol Metab 2015;100: E632-E637.

18 Sozopoulos E, Litsiou H, Voutsinas G, et al. Mutational and immunohistochemical study of the PI3K/Akt pathway in papillary thyroid carcinoma in Greece. Endocr Pathol 2010;21:90-100.

19 Oishi N, Kondo T, Nakazawa T, et al. Frequent BRAFV600E and absence of TERT promoter mutations characterize sporadic pediatric papillary thyroid carcinomas in Japan. Endocr Pathol 2017;28:103-111.

20 Mochizuki K, Kondo T, Nakazawa T, et al. RET rearrangements and BRAF mutation in undifferentiated thyroid carcinomas having papillary carcinoma components. Histopathology 2010;57:444-450.

21 Ragazzi M, Ciarrocchi A, Sancisi V, et al. Update on anaplastic thyroid carcinoma: morphological, molecular, and genetic features of the most aggressive thyroid cancer. Int J Endocrinol 2014;2014:1-13.

22 Kondo T, Nakazawa T, Ma D, et al. Epigenetic silencing of TTF-1/NKX2-1 through DNA hypermethylation and histone $\mathrm{H} 3$ modulation in thyroid carcinomas. Lab Invest 2009;89:791-799.

23 Takano T, Ito Y, Hirokawa M, et al. BRAF V600E mutation in anaplastic thyroid carcinomas and their accompanying differentiated carcinomas. Br J Cancer 2007;96:1549-1553.

24 Hunt JL, Tometsko M, LiVolsi VA, et al. Molecular evidence of anaplastic transformation in coexisting well-differentiated and anaplastic carcinomas of the thyroid. Am J Surg Pathol 2003;27:1559-1564.

25 Nikiforov YE. Thyroid carcinoma: molecular pathways and therapeutic targets. Mod Pathol 2008;21:S37-S43.

26 Xing M. Molecular pathogenesis and mechanisms of thyroid cancer. Nat Rev Cancer 2013;13:184-199.

27 Kondo T, Ezzat S, Asa SL. Pathogenetic mechanisms in thyroid follicular-cell neoplasia. Nat Rev Cancer 2006;6:292-306.
28 Nikiforova MN, Kimura ET, Gandhi M, et al. BRAF mutations in thyroid tumors are restricted to papillary carcinomas and anaplastic or poorly differentiated carcinomas arising from papillary carcinomas. J Clin Endocrinol Metab 2003;88:5399-5404.

29 Melo M, da Rocha AG, Vinagre J, et al. TERT promoter mutations are a major indicator of poor outcome in differentiated thyroid carcinomas. J Clin Endocrinol Metab 2014;99:E754-E765.

30 Liu T, Wang N, Cao J, et al. The age- and shorter telomere-dependent TERT promoter mutation in follicular thyroid cell-derived carcinomas. Oncogene 2014; 33:4978-4984.

31 Salvatore G, Nappi TC, Salerno P, et al. A cell proliferation and chromosomal instability signature in anaplastic thyroid carcinoma. Cancer Res 2007;67: 10148-10158.

32 Xing M, Alzahrani AS, Carson KA, et al. Association between BRAF V600E mutation and mortality in patients with papillary thyroid cancer. JAMA 2013;309: 1493-1501.

33 Lim JY, Hong SW, Lee YS, et al. Clinicopathologic implications of the BRAF(V600E) mutation in papillary thyroid cancer: a subgroup analysis of 3130 cases in a single center. Thyroid 2013;23:1423-1430.

34 Liu R, Bishop J, Zhu G, et al. Mortality risk stratification by combining BRAF V600E and TERT promoter mutations in papillary thyroid cancer. JAMA Oncol 2017;3:202-208.

35 Gandolfi G, de Biase D, Sancisi V, et al. Deep sequencing of KIT, MET, PIK3CA, and PTEN hotspots in papillary thyroid carcinomas with distant metastases. Endocr Relat Cancer 2014;21:L23-L26.

36 Charles R-P, Silva J, Iezza G, et al. Activating BRAF and PIK3CA mutations cooperate to promote anaplastic thyroid carcinogenesis. Mol Cancer Res 2014;12: 979-986.

37 Oike $\mathrm{T}$, Ogiwara $\mathrm{H}$, Nakano $\mathrm{T}$, et al. Inactivating mutations in SWI/SNF chromatin remodeling genes in human cancer. Jpn J Clin Oncol 2013;43:849-855.

38 Yoshimoto T, Matsubara D, Nakano T, et al. Frequent loss of the expression of multiple subunits of the SWI/ SNF complex in large cell carcinoma and pleomorphic carcinoma of the lung. Pathol Int 2015;65:595-602.

39 Wiegand $\mathrm{KC}$, Lee AF, Al-Agha OM, et al. Loss of BAF250a (ARID1A) is frequent in high-grade endometrial carcinomas. J Pathol 2011;224:328-333.

40 Hohmann AF, Vakoc CR. A rationale to target the SWI/ SNF complex for cancer therapy. Trends Genet 2014;30:356-363.

41 Pita JM, Figueiredo IF, Moura MM, et al. Cell cycle deregulation and TP53 and RAS mutations are major events in poorly differentiated and undifferentiated thyroid carcinomas. J Clin Endocrinol Metab 2014;99: E497-E507.

42 McFadden DG, Vernon A, Santiago PM, et al. p53 constrains progression to anaplastic thyroid carcinoma in a Braf-mutant mouse model of papillary thyroid cancer. Proc Natl Acad Sci USA 2014;111:E1600-E1609.

Supplementary Information accompanies the paper on Modern Pathology website (http://www.nature.com/ modpathol) 